\title{
CORTISOL LEVELS AND AUTONOMIC CONTROL OF HEART RATE IN HEALTHY SUBJECTS
}

\section{Milana Drumond Ramos Santana ${ }^{1,2,5}$, Eli Carlos Martiniano ${ }^{2}$, LaRissa Raylane Lucas Monteiro ${ }^{2}$, Maria do Socorro Santos De Oliveira ${ }^{2}$, Vitor E. Valenti ${ }^{3}$, David M. Garner ${ }^{4}$, Franciele Margues VANDERLEI $^{3}$, LUIZ CARLOS DE ABREU ${ }^{1,5}$}

\author{
${ }^{1}$ Laboratory of Study Design and Scientific Writing, Faculdade de Medicina do ABC, Santo André, SP, Brazil. \\ ${ }^{2}$ Faculty of Juazeiro do Norte, Juazeiro do Norte, CE, Brazil \\ ${ }^{3}$ Post-Graduate Program in Physiotherapy, UNESP, Presidente Prudente, SP, Brazil. \\ ${ }^{4}$ Cardiorespiratory Research Group, Department of Biological and Medical Sciences, Faculty of Health and Life \\ Sciences, Oxford Brookes University, Gipsy Lane, Oxford OX3 OBP, United Kingdom \\ ${ }^{5}$ Post-Doctoral Program of the Faculty of Medicine of ABC, Santo André, SP, Brazil.
}

\begin{abstract}
Introduction: There is an increase in level of stress in the general population because of the social, personal and professional demands. Currently, there are only simple tools that can safely measure this stress such as levels of cortisol and heart rate variability (HRV).

Objective: To analyze the relationship between salivary cortisol and the cardiac autonomic modulation. Methods: A total of fifty-one male and female subjects between 18 and 40 years old were evaluated. Saliva collection was achieved for the salivary cortisol dosage. The collection was performed through the Salivette ${ }^{\circledR}$ tube. After this collection, the median cortisol levels $(0.24 \mathrm{ug} / \mathrm{dl})$ were analyzed and the volunteers were divided into two groups: i) cortisol below the mediane ii) cortisol above the median. After this division, each group consisted of 25 volunteers and then was verified the following information: age, gender, weight, height, body mass index (BMI), blood pressure. Shortly thereafter was assessment of cardiac autonomic modulation por meio da HRV. The Polar RS800cx heart rate receiver was placed on the chest of the volunteers, in the vicinity of the distal third of the sternum. The volunteers were instructed to remain in rest with spontaneous breathing in dorsal position for 20 minutes. HRV analysis included geometric, time and frequency domain indices.

Results: There were no statistical differences for the two groups regarding systolic and diastolic blood pressure, heart rate, RR intervals or linear and frequency indices for the volunteers. In addition, also there was no correlation the cortisol with the analyzed variables (SAP, $\mathrm{p}=0.460$; DAP, $\mathrm{p}=0.270 ; \mathrm{HR}, \mathrm{p}=0.360 ; \mathrm{RR}, \mathrm{p}=0.380 ; \mathrm{SDNN}, \mathrm{p}=0.510$;

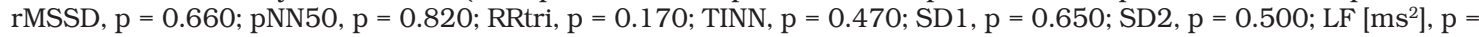
0.880; LF [nu], p = 0.970; HF [ms ${ }^{2}$ ], p = 0.870; HF [nu], p = 0.960; LF/HF, p = 0.380

Conclusion: Heart rate variability autonomic control was unchanged in healthy subjects with physiological distribution of salivary cortisol levels. There was no association between normal salivary cortisol and resting autonomic regulation of heart rate.
\end{abstract}

Key words: cardiovascular system; autonomic nervous system, salivary cortisol.

\section{INTRODUCTION}

Contemporary lifestyles generate a high level of psychological stress that causes marked subjective and biological responses (1). In chronic cases, it can initiate deficiencies in physical and mental health $(2,3)$, and increase the biological aging processes $(3)$.

Contrary to this background, there has been an increase of the psychological disorders such as anxiety and depression, and these disorders are associated with increased cardiovascular risk (4). Deviations in homeostasis of autonomic function with an imbalance between the sympathetic and parasympathetic autonomic nervous systems could be reasons involved in this increased cardiovascular risk $(5,6)$.

Milana Drumond Ramos Santana, Faculdade de Juazeiro do Norte. Rua São Francisco, 1224, Juazeiro do Norte, CE, Brazil. Phone/Fax: +55 (88) 2101-2777. E-mail: mildrumond@ hotmail.com 
Physical or emotional stress stimulates the hypothalamus to synthesize corticotrophin releasing hormone (CRH), which thereby stimulates the pituitary gland to synthesize the adrenocorticotropic hormone (ACTH), which permeates through the bloodstream to the adrenal glands and stimulates the synthesis of cortisol (7). Concurrently, activation of the sympathetic nervous system occurs and catecholamine's (noradrenaline and adrenaline) are released, which activate an increase in the frequency and strength of cardiac contraction, cardiac output and blood pressure (8). Cortisol is the primary effector hormone of the hypothalamic-pituitaryadrenal axis stress response system (9).

Thus, a technique that has been revealed to be effective in measuring stress and anxiety is the quantification of cortisol in saliva. As there is a direct relationship between the levels of some hormones and medications in saliva and blood, the salivary cortisol dosage has been applied owing to the simplicity in sample collection and the sensitivity of the technique (7).

The regulation of the cardiac function, stimulated by the autonomic nervous system (ANS), allows us to evaluate the imbalance between the activity of the sympathetic and parasympathetic responses. With regards to the heart, the ANS interferes with cardiac frequency and contractility. In addition to increasing or decreasing heart rate, the autonomic system also hinders the regularity between heart beats. This variation in the intervals between one heart beat and another is referred to as the heart rate variability (HRV) $(10,11)$.

HRV has emerged as a simple, inexpensive, reliable and non-invasive measure of autonomic impulses. It represents one of the most promising quantitative markers of autonomic balance (10). Power spectral analysis of RR interval variability has been widely applied to assess cardiac autonomic modulation (12).

Consequently, since there is now an elevation in levels of stress in the population due to social, personal and professional strains these are simple markers that can securely measure these stresses - cortisol levels and HRV. The objective of this study was to verify the association between salivary cortisol and autonomic cardiac regulation. It is speculated that there is a relationship between the two measures since cortisol is the primary effector hormone of the stress response system.

\section{METHODS}

\section{Study population}

We evaluated a total of 50 healthy subjects ( 25 men) aged 18 to 40 years old. All subjects were informed about the study's procedures and objectives and, after agreeing, confidentially signed an informed consent form. The median salivary cortisol level was calculated $(0.24 \mathrm{ug} / \mathrm{dl})$ and the subjects were divided into two groups: G1 - cortisol below $0.24 \mathrm{ug} / \mathrm{dl}(\mathrm{n}=25)$ and G2 cortisol above $0.24 \mathrm{ug} / \mathrm{dl}(\mathrm{n}=25)$. The research was approved by the Research Ethics Committee in Research (Number 993.350).

\section{Non-inclusion Criteria}

We excluded smokers, subjects with cardiorespiratory, metabolic or neurological disorders that prevented the subjects from performing the procedures, in addition to treatment with medications that influence cardiac autonomic regulation. Also, women at 10-15 days and 20-25 days after the first day of the menstrual cycle and pregnant women so as to circumvent influence of the luteal and follicular phase of the menstrual cycle.

\section{Experimental Protocols}

Data collection was performed in a laboratory with the temperature between $21^{\circ} \mathrm{C}$ and $25^{\circ} \mathrm{C}$, humidity amid $50 \%$ and $60 \%$ and time fixed between 8:00 am and 12:00 am to evade circadian cycle effects. Volunteers were instructed not to ingest alcoholic beverages, caffeine or other ANS stimulants in the 24 hours before the evaluation and to remain silent during the data collection. The following information was registered: age [years], gender [masculine/feminine], weight $[\mathrm{kg}]$, height [meters] and body mass index (BMI) $\left[\mathrm{kg} / \mathrm{m}^{2}\right]$. 


\section{Cortisol Levels Measurement}

The subjects were instructed to avoid eating and drinking one hour before collecting the saliva and washed their oral cavity with water. The Salivette ${ }^{\circledR}$ tubes were stored under refrigeration until material analysis.

The collection was performed through the Salivette ${ }^{\circledR}$ tube, consisting of a plastic tube containing a cotton roller of high absorption and kept in the oral cavity for about three minutes and then placed in the holder inside the plastic tube collector. Later, after collection of all samples the Salivette ${ }^{\circledR}$ tube was centrifuged for 20 minutes at $2800 \mathrm{rpm}$ and at a temperature of $4{ }^{\circ} \mathrm{C}$. During centrifugation, the saliva passed from the cylindrical shape of the swab through the cavity at the bottom of the suspended tube to the clean centrifuge tube. Secretion and suspended particles were located at the conical tip of the tube, to allow easy decanting of the clarified saliva.

Following centrifugation, saliva was transferred to a capped eppendorff tube and remained in a freezer at $-20{ }^{\circ} \mathrm{C}$ until the time of the assay. During the transportation phase, the material was chilled by the use of dry ice, Styrofoam and thermal bag. The samples were processed using the electro-chemiluminescence method.

\section{Blood pressure measurement}

The blood pressure was measured using the auscultatory method, via an aneroid sphygmomanometer $\left(\operatorname{Tycos}^{\circledR}\right)$ and stethoscope $\left(\right.$ Sprague $\left.{ }^{\circledR}\right)$. The values of systolic blood pressure and diastolic blood pressure were also recorded. The values of systolic blood pressure of at most $140 \mathrm{mmHg}$ and diastolic of $90 \mathrm{mmHg}$ were considered to avoid a potential effect of arterial hypertension on the results.

\section{HRV analysis}

During RR intervals recording the subjects remained 20 minutes at seated rest under spontaneous breathing. We used a 10 minutes for stabilization of autonomic balance.

HRV analysis was performed according to directives from the Task Force publication ${ }^{(13)}$. HRV was recorded using the Polar RS800cx chest strap measuring device (Polar Electro Oy, Kempele, Finland) which was validated to capture beat-to-beat heart rate (HR) ${ }^{(14)}$. Particulars of HRV analysis were specified in the cited studies ${ }^{(15)}$. HRV analysis included geometric, time and frequency domain indices which were computed by Kubios HRV ${ }^{\circledR}$ analysis software.

The Polar transmitter detects all heart beats through electrical signals in the left ventricle muscle and the data recorded transmit the signal to the computer with an infrared approach through a wireless technology. The software enabled the visualization of HR and the extraction of a cardiac period (RR interval) file in "txt" format.

Digital filtering was undertaken to remove artifacts. The filtering process method in the software included an algorithm that calculates median and moving average based filtering methods to detect errors. The algorithm calculated several more matching 'candidates' to replace the identified errors. Before elaborating the preview curve, which presents the optional corrections, the algorithm verifies the difference between consecutive accepted RR intervals and makes the series of corrected values (typically 2-4 intervals) to follow identical differential coefficient. The error correction algorithm precisely preserves the total time of the recording, i.e. the sum of RR intervals is exactly the same as the elapsed time. The minimum protection zone used was six beats per minute (http://support.polar.com/en/ support/tips/How_R-R_Data_is_ Filtered).

Following digital filtering supplemented with manual filtering for the elimination of artifacts, 256 stable RR intervals were necessary for the data analysis. Only series with more than $95 \%$ sinus rhythm was included in the study (13).

The time domain analysis was performed in terms of SDNN (standard deviation of normal-to normal RR intervals), pNN50 (percentage of adjacent RR intervals with a difference of duration greater than $50 \mathrm{~ms}$ ) and RMSSD (root-mean square of differences between adjacent normal RR intervals in a time interval) (Table 1) (13). 
To obtain the spectral indexes for HRV analysis in the frequency domain, the frequency recordings underwent mathematical processing, generating a tachogram that expressed the variation of RR intervals as a function of time. The tachogram contained a signal apparently periodic that varies in time and was processed by the mathematical Fast Fourier Transform algorithms (13).

Low frequency (LF- ranging between 0.04 and $0.15 \mathrm{~Hz}$ ) and high frequency (HF- ranging from 0.15 to $0.4 \mathrm{~Hz}$ ) spectral components were appointed in normalized units (n.u.). The ratio between these components in absolute values (LF/HF) represents the relative value of each spectral component in relation to the total potential minus the very low frequency (VLF) components (13).

The triangular index (RRtri) was calculated from the construction of a density histogram of RR intervals, which contains the horizontal axis of all possible RR intervals measured on a discrete scale with $7.8125 \mathrm{~ms}$ boxes (1/128 seconds) and on the vertical axis, the frequency with which each occurred. The union of points of the histogram columns forms a triangle-like shape. The RRtri was obtained by dividing the number of RR intervals used to construct the histogram by their modal frequency (i.e., the RR interval that most frequently appeared on RR).

The triangular interpolation (TINN) consists of the measure of the base of a triangle. The method of least squares is used to determine the triangle. The RRtri and the TINN express the overall variability of the RR intervals (14).

The Poincaré plot is a map of points in Cartesian coordinates that is constructed from the values of the RR intervals. Each point is represented on the $\mathrm{x}$-axis by the previous normal RR interval and on the $\mathrm{y}$-axis by the following RR interval.

For the quantitative analysis of the plot, an ellipse was fitted to the points of the chart, with the center determined by the average RR interval. The standard deviation of the instantaneous variability of the beat-to-beat heart rate (SD1) indices were calculated to measure the standard deviation of the distances of the points from the diagonal $\mathrm{y}=\mathrm{x}$, and the standard deviation of long-term continuous RR interval variability (SD2) measures the standard deviation of the distances of points from the line $y=-x+R R m$, where $R R m$ is the average RR interval. The SD 1 is an index of the instantaneous recording of the variability of beat-to-beat and represents the parasympathetic activity, whereas the SD2 index represents the long-term HRV and reflects the overall variability (14).

For calculation of the linear indices we used the HRV Analysis software (Kubios HRV v.1.1 for Windows, Biomedical Signal Analysis Group, Department of Applied Physics, University of Kuopio, Finland).

\section{Statistical analysis}

Sample size calculation was based on a previous publication (16), which considered the at rest rMSSD index. The size of the difference was assumed for $11 \mathrm{~ms}$, considering a standard deviation of $16.2 \mathrm{~ms}$, with alpha risk of $5 \%$ and beta risk of $80 \%$. So, a minimum of 18 subjects were specified.

Means and standard deviations were computed with normal Gaussian distribution of the data evaluated through the Shapiro-Wilk goodness-of-fit test ( $z$ value $>1.0$ ). To equate variables between groups we applied non-paired Student t-test for parametric distributions and MannWhitney for nonparametric distributions. Differences were considered significant when the probability of a Type I error was less than $5 \%(\mathrm{p}<0.05)$.

To assess the magnitude of differences between groups we computed the effect size using Cohen's d for significant differences. Large effect magnitude was considered for values $\geq 0.9$, medium for values between 0.5 and 0.9 and small for values $\leq 0.5$ (17).

\section{RESULTS}

Table 1 illustrates the mean and standard deviation of the variables of age, height, mass and BMI of the volunteers. 
$\begin{array}{lllllllllllllllllllllllllllllllllll}\text { A } & \text { C } & \text { T } & \text { A } & & \text { M } & \text { E } & \text { D } & \text { I } & \text { C } & \text { A } & \text { M } & \text { A } & \text { R } & \text { T } & \text { I } & \text { N } & \text { I } & \text { A } & \text { N } & \text { A } & & 2 & 0 & 1 & 7 & & 17 / 2\end{array}$

Table 1. Age, height, weight and BMI of the volunteers.

\begin{tabular}{|c|c|c|c|c|}
\hline Variables & G1 (n=25) & G2 $(\mathbf{n}=\mathbf{2 5})$ & $p$ value & Cohen's d \\
\hline Age (years) & $28.00 \pm 6.97$ & $29.48 \pm 6.82$ & 0.4518 & 0.21 \\
\hline Height $(\mathrm{m})$ & $1.58 \pm 0.08$ & $1.62 \pm 0.08$ & 0.0722 & 0.50 \\
\hline Weight $(\mathrm{kg})$ & $62.64 \pm 11.10$ & $66.55 \pm 11.07$ & 0.2188 & 0.35 \\
\hline BMI $\left(\mathrm{kg} / \mathrm{m}^{2}\right)$ & $25.79 \pm 6.31$ & $25.18 \pm 4.21$ & 0.9073 & 0.11 \\
\hline
\end{tabular}

Legend: Mean \pm Standard-deviation; G1: cortisol below $0.24 \mathrm{ug} / \mathrm{dl}$; G2: cortisol above 0.24 ug/dl; m: meters; kg: kilograms; BMI: height and body mass index; $\mathrm{kg} / \mathrm{m}^{2}$ : kilograms per square meter.

Table 2 illustrates the mean and standard deviation of systolic (SBP) and diastolic blood pressure (DBP), heart rate (HR), RR intervals, geometric, time and frequency domain indices of HRV. As predicted, cortisol levels were significantly higher in the G2, however, we found no significant difference regarding SBP, DBP and HRV between groups.

Table 2. Mean and standard deviation of salivary cortisol levels, SBP, DBP, HR, RR intervals, time and frequency domain of HRV.

\begin{tabular}{|l|c|c|c|c|}
\hline Variables & G1 (n=25) & G2 (n=25) & $p$ value & Cohen's d \\
\hline Cortisol (ug/dl) & $0.14 \pm 0.05$ & $0.37 \pm 0.13$ & $<0.0001$ & 2.33 \\
\hline SBP $(\mathrm{mmHg})$ & $112.80 \pm 12.42$ & $120.80 \pm 18.69$ & 0.1539 & 0.50 \\
\hline DBP (mmHg) & $76.40 \pm 8.10$ & $80.40 \pm 12.74$ & 0.3512 & 0.37 \\
\hline HR (bpm) & $90.04 \pm 17.99$ & $83.29 \pm 17.09$ & 0.2003 & 0.38 \\
\hline RR (ms) & $689.62 \pm 122.52$ & $748.68 \pm 136.29$ & 0.1137 & 0.45 \\
\hline SDNN (ms) & $39.92 \pm 16.57$ & $44.60 \pm 15.17$ & 0.3038 & 0.29 \\
\hline rMSSD (ms) & $27.53 \pm 14.59$ & $34.45 \pm 20.21$ & 0.2072 & 0.39 \\
\hline NN50 (ms) & $17.24 \pm 36.39$ & $15.10 \pm 16.51$ & 0.1174 & 0.07 \\
\hline pNN50 (ms) & $10.56 \pm 12.90$ & $15.86 \pm 18.49$ & 0.2101 & 0.33 \\
\hline RRtri (ms) & $8.03 \pm 4.83$ & $9.64 \pm 3.89$ & 0.1743 & 0.36 \\
\hline TINN (ms) & $156.60 \pm 64.23$ & $174.00 \pm 67.22$ & 0.3541 & 0.26 \\
\hline SD1 (ms) & $19.59 \pm 10.38$ & $24.49 \pm 14.35$ & 0.2072 & 0.39 \\
\hline SD2 (ms) & $52.18 \pm 22.96$ & $57.25 \pm 18.93$ & 0.3988 & 0.24 \\
\hline LF (ms $\left.{ }^{2}\right)$ & $524.96 \pm 481.42$ & $663.31 \pm 530.92$ & 0.9381 & 0.27 \\
\hline LF (nu) & $61.63 \pm 19.02$ & $60.41 \pm 18.40$ & 0.8193 & 0.06 \\
\hline HF (ms $\left.{ }^{2}\right)$ & $339.45 \pm 424.65$ & $529.68 \pm 570.74$ & 0.1138 & 0.37 \\
\hline HF (nu) & $38.16 \pm 18.93$ & $39.34 \pm 18.33$ & 0.8244 & 0.06 \\
\hline LF/HF & $2.95 \pm 3.70$ & $2.38 \pm 2.50$ & 1.000 & 0.18 \\
\hline
\end{tabular}


Legend: ms: milliseconds; bpm: beat per minute; mmHg: millimeters of mercury; ug/dl: micrograms per deciliter; nu: normalized unit; $\mathrm{ms}^{2}$ : absolute unity; SBP: systolic blood pressure; DBP: diastolic blood pressure; HR: heart rate; SDNN: standard deviation of all normal RR intervals; pNN50: percentage of adjacent RR intervals lasting difference greater than 50 milliseconds; rMSSD: square root of the mean square of differences between adjacent normal RR intervals; LF: low frequency; HF: high frequency; LF/HF: ratio low frequency/high frequency; TINN: triangular interpolation of NN interval histogram; RRtri: triangular index; SD1: standard deviation of the instantaneous variability of the beat-to-beat heart rate; SD2: standard deviation of long-term continuous RR interval variability.

We also preformed correlation analysis between cortisol levels and resting HRV in the 50 subjects. There was no significant correlation between the variables (Table 3). Considering that we found no significant correlation between variables it was superfluous to perform a linear regression.

Table 3. Correlation of cortisol with SBP, DBP, HR, RR intervals, time and frequency domain of HRV.

\begin{tabular}{|l|c|c|}
\hline Variables & $\mathbf{R}$ & $\boldsymbol{p}$ value \\
\hline SAP $(\mathrm{mmHg})$ & 0.080 & 0.460 \\
\hline DAP $(\mathrm{mmHg})$ & 0.120 & 0.270 \\
\hline HR (bpm) & 0.10 & 0.360 \\
\hline RR (ms) & -0.10 & 0.380 \\
\hline SDNN (ms) & 0.070 & 0.510 \\
\hline rMSSD (ms) & 0.050 & 0.660 \\
\hline pNN50 (ms) & 0.020 & 0.820 \\
\hline RRtri (ms) & 0.160 & 0.170 \\
\hline TINN (ms) & 0.080 & 0.470 \\
\hline SD1 (ms) & 0.050 & 0.650 \\
\hline SD2 (ms) & 0.070 & 0.500 \\
\hline LF (ms $\left.{ }^{2}\right)$ & 0.010 & 0.880 \\
\hline LF (nu) & 0.003 & 0.970 \\
\hline HF $\left(m s^{2}\right)$ & -0.010 & 0.870 \\
\hline HF $(n u)$ & -0.004 & 0.960 \\
\hline LF $/ H F$ & -0.100 & 0.380 \\
\hline
\end{tabular}

Legend: ms: milliseconds; bpm: beat per minute; mmHg: millimeters of mercury; nu: normalized unit; $\mathrm{ms}^{2}$ : absolute unity; SBP: systolic blood pressure; DBP: diastolic blood pressure; HR: heart rate; SDNN: standard deviation of all normal RR intervals; pNN50: percentage of adjacent RR intervals lasting difference greater than 50 milliseconds; rMSSD: square root of the mean square of differences between adjacent normal RR intervals; LF: low frequency; HF: high frequency; LF/HF: ratio low frequency/high frequency; TINN: triangular interpolation of NN interval histogram; RRtri: triangular index; SD 1: standard deviation of the instantaneous variability of the beat-to-beat heart rate; SD2: standard deviation of long-term continuous RR interval variability. 


\section{DISCUSSION}

Considering that the ANS and Hypothalamic-Pituitary-Adrenal axis is physically connected and synchronized (18), which is demonstrated in physiologically stressful situations (19), we sought to evaluate this association in healthy subjects. Nevertheless, we failed to find a connection between salivary cortisol levels and resting HRV.

In order to extend the results, we decided to split healthy subjects into two equal groups according to median baseline salivary cortisol levels (higher or lower than $0.24 \mathrm{ug} / \mathrm{dl}$ ). There was a significant change between the cortisol value of the two groups ( $p<0.0001)$. However, it was detected that the cardiac autonomic modulation continued without significant alteration.

The ANS and the Hypothalamic-Hypophysis Adrenal (HHA) axis control important physiological functions of the human body. Fluctuations of the function in these two systems are associated with metabolic disorders and dysregulation of the cardiovascular system, with altered autonomic cardiac function (20,21). Evaluation of the ANS function through HRV and the use of salivary biomarkers such as salivary cortisol to assess the purpose of the ANS and HHA axis can help identify patterns associated with the stage of evolution of some diseases, beyond the severity and effectiveness of treatment $(21,22)$.

There is an absence of notable scientific literature highlighting the influence of cortisol levels, performing as a stressor factor, and autonomic cardiac modulation. Most of the studies in the relevant research literature evaluate some motor/psychological and/or pathology to detect an association between psychological stress and the ANS. Clays et al. (23) is a study that correlated the perception of psychological stress in an occupational environment with the parasympathetic modulation of 653 healthy male workers, illustrating that the higher this perception, the lower the parasympathetic modulation.

Studies show that low HRV, indicating a sympathetic predominance, is related to specific stress patterns and, consequently, to higher values of cortisol. Chida \& Steptoe (24) performed a systematic review with meta-analysis to identify cortisol responses with psychosocial factors. The authors (24) found that cortisol increase after awake was positively associated with stress at work and overall stress of life and negatively with fatigue, burnout, and exhaustion. Therefore, the authors (24) concluded that different psychosocial factors are associated with an increased or decreased cortisol response.

Some studies have examined the relationship between HRV and cortisol. High levels of cortisol were associated with low HF and LF values, but showed no change in HF/LF in a cross-sectional study ${ }^{(25)}$. However, as in the results of the present study, results were also published that showed no association between HRV and cortisol $(26,27)$.

In addition to cross-sectional studies, there are studies in the literature that provoke stress reactions to evaluate the association of HRV and cortisol. Sgoifo et al. (28) investigated the relationship between autonomic cardiac, endocrine and behavioral responses to real-life stress episodes. This authors (28) exposed 30 college students to two stressful social challenges and found that individuals who had higher levels of sympathetic predominance also had increases in cortisol levels. Therefore, the results suggest that there is a narrow relationship between the degree of physiological excitability and the style of behavioral adaptation to social stressors. It is worth noting that in the present study no intervention was performed that could cause stress in the individual, and therefore, this fact may explain, at least in parts, the absence of correlation between HRV and cortisol.

Cortisol is recognized as a "stress hormone" formed by the glucocorticoid that is released by the adrenal glands when the Hypothalamic-Pituitary Adrenal (HPA) axis is activated by psychological stress (29). Cortisol is vital for establishing homeostasis after exposure to a stressor (29). According to Castro and Moreira (30), cortisol circulates in the bloodstream connected to the transporter proteins, transcortin, the corticoid transporter protein (CBG), and albumin. Only $5 \%$ to $10 \%$ is in the free form; the biologically active element of the hormone.

Nevertheless, measurement of cortisol levels in saliva is gaining extensive acceptance as a diagnostic technique. The salivary cortisol test has been applied for HPA axis function, under 
various cognitive conditions and in the presence of mental stress and anxiety (31). Previously, when an individual is exposed to the stressful situation, the neuroendocrine systems are activated and secrete substances such as adrenaline and cortisol, promoting physiological reactions in response to stress (32).

Regarding the effects of mental stress, anxiety and depression on the modulation of the ANS, Langewitz et al. (33) evaluated the effect of psychological stress on the ANS by means of HRV, where decreases in some HRV variables were observed. According to Morino et al. (34) HRV spectral analysis is beneficial in assessing psychological tension and anxiety.

Statistically there was no relationship between changes in cortisol and cardiac autonomic regulation. Yet, when these parameters were analyzed in detail, it was perceived that some of them responded with a greater influence in relation to others, for instance, HF indices in absolute units, NN50 and RR with p value of approximately 0.11 . Another statistically trivial change is the SBP where in the group with cortisol levels above the median, values are greater in relation to the group with cortisol levels below the median.

These results allow us to reflect why these changes in salivary cortisol levels did not significantly influence the cardiac autonomic control process. A potential theory for such a discovery would be that the ANS suffers considerable influence of the hormone cortisol only in situations where more intense stress occurs to the organism, as pathological conditions, psychological tests and the performance of strenuous physical exercises.

The present study contributes to the scarce literature that addresses the relationship between HRV and cortisol. The strength of the study is the standardization of HRV measurements with well-established criteria and filtering processing that ensures study quality. However, some limitations of the study should be highlighted. Concerning the methodological aspects, the studied age range was wide (18 to 40 years), yet, prioritizing the exclusion criteria, we sought normative standards in relation to the selection sample. Additionally, it should be recognized that the sample consisted of both males and females and individuals classified as overweight according to the World Health Organization.

It is worth highlighting that this current study is significant because it illustrates discontinuities between the association of cortisol quantities and cardiac autonomic modulation in relation to the primary effector response of the system that directs psychological stress in situations where there is no influence of a stressor factor.

\section{CONCLUSION}

Heart rate variability autonomic control was unchanged in healthy subjects with physiological distribution of salivary cortisol levels. There was no association between normal salivary cortisol and resting autonomic regulation of heart rate.

\section{REFERENCES}

1. Dickerson SS, Gruenewald TL, Kemeny ME. When the social self is threatened: shame, physiology, and health. J Pers 2004; 72(6): 1191-216.

2. Dickerson SS, Gruenewald TL, Kemeny ME. Psychobiological responses to social self threat: functional or detrimental? Self and Identity 2009; 8(2-3): 270-85.

3. Miller GE, Chen E, Parker KJ. Psychological stress in childhood and susceptibility to the chronic diseases of aging: moving toward a model of behavioral and biological mechanisms. Psychol Bull 2011; 137(6): 959-97.

4. Munakata M, Ichi S, Nunokawa T, et al. Influence of night shift work on psychologic state and cardiovascular and neuroendocrine responses in healthy nurses. Hypertens Res 2001; 24(1): 25-31.

5. Kubzansky LD, Kawachi I. Going to the heart of the matter: do negative emotions cause coronary heart disease? J Psychosom Res 2000; 48(4-5): 323-37. 
6. Longhi A, Tomaz CAB. Variabilidade da frequência cardíaca, depressão, ansiedade e estresse em intensivistas. Rev Bras Cardiol 2010; 23(6): 315-23.

7. Akyuz S, Pince S, Hekim N. Children's stress during a restorative dental treatment: assessment using salivary cortisol measurements. J Clin Pediatr Dent 1996; 20(3): 219-23.

8. Aubert AE, Seps B, Beckers F. Heart rate variability in athletes. Sports Med 2003; 33(12): 889-919.

9. O'Connor DB, Green JA, Ferguson E, O'Carroll RE, O'Connor RC. Cortisol reactivity and suicidal behavior: investigating the role of hypothalamic-pituitary-adrenal axis responses to stress in suicide attempters and ideators. Psychoneuroendocrinology 2017; 75: 183-91.

10. Vanderlei LCM, Pastre CM, Hoshi RA, Carvalho TD, Godoy MF. Basic notions of heart rate variability and its clinical applicability. Rev Bras Cir Cardiovasc 2009; 24(2): 205-17.

11. Santana MD, Martiniano, EC, Monteiro LR, et al. Musical auditory stimulation influences heart rate autonomic responses to endodontic treatment. Evid Based Complement Alternat Med 2017; 2017: 4847869.

12. Miura K, Matsumura K, Nakamura Y, Kurokava H, Kajiyama M, Takata Y. Suppression of cardiac sympathetic nervous system during dental surgery in hypertensive patients. Hypertens Res 2000; 23(3): 207-12.

13. Task Force of the European Society of Cardiology and the North American Society of Pacing and Electrophysiology. Heart rate variability: standards of measurement, physiological interpretation and clinical use. Circulation 1996; 93(5): 1043-65.

14. Barbosa MP, Silva NT, Azevedo FM, Pastre CM, Vanderlei LC. Comparison of Polar ${ }^{\circledR}$ RS800G3 ${ }^{\mathrm{TM}}$ heart rate monitor with Polar ${ }^{\circledR} \mathrm{S} 810 \mathrm{i}^{\mathrm{TM}}$ and electrocardiogram to obtain the series of RR intervals and analysis of heart rate variability at rest. Clin Physiol Funct Imaging 2016; 36(2): 112-7.

15. Carvalho TD, Pastre CM, Rossi RC, Abreu LC, Valenti VE, Vanderlei LCM. Geometric index of heart rate variability in chronic obstructive pulmonary disease. Rev Port Pneumol. 2011;17(6):260-5.

16. Vanderlei FM, Moreno IL, Vanderlei LCM, Pastre CM, Abreu LC, Ferreira C. Comparison of the effect of hydration with water or isotonic solution on the recovery of cardiac autonomic modulation. Int $\mathrm{J}$ Sport Nutr Exerc Metab 2015; 25(2): 145-53.

17. Quintana DS. Statistical considerations for reporting and planning heart rate variability case-control studies. Psychophysiology 2017; 54(3): 344-9.

18. Lovell B, Moss M, Wetherell MA. Perceived stress, common health complaints and diurnal patterns of cortisol secretion in young, otherwise healthy individuals. Horm Behav 2011; 60(3): 301-5.

19. Jensen MA, Garde AH, Kristiansen J, Nabe-Nielsen K, Hansen ÅM. The effect of the number of consecutive night shifts on diurnal rhythms in cortisol, melatonin and heart rate variability (HRV): a systematic review of field studies. Int Arch Occup Environ Health 2016; 89(4): 531-45.

20. Licht CM, Vreeburg SA, van Reedt Dortland AK, et al. Increased sympathetic and decreased parasympathetic activity rather than changes in hypothalamic-pituitary-adrenal axis activity is associated with metabolic abnormalities. J Clin Endocrinol Metab 2010; 95(5): 2458-66.

21. Marques AH, Silverman MN, Sternberg EM. Evaluation of stress systems by applying noninvasive methodologies: measurements of neuroimmune biomarkers in the sweat, heart rate variability and salivary cortisol. Neuroimmunomodulation 2010; 17(3): 205-8.

22. Nater UM, Rohleder N. Salivary alpha-amylase as a non-invasive biomarker for the sympathetic nervous system: current state of research. Psychoneuroendocrinology 2009; 34(4): 486-96.

23. Clays E, De Bacquer D, Crasset V, et al. The perception of work stressors is related to reduced parasympathetic activity. Int Arch Occup Environ Health 2011; 84(2): 185-91.

24. Chida Y, Steptoe A. Cortisol awakening response and psychosocial factors: A systematic review and meta-analysis. Biol Psychol 2009; 80(3): 265-78.

25. Stalder T, Evans P, Hucklebridge F, Clow A. Associations between the cortisol awakening response and heart rate variability. Psychoneuroendocrinology 2011; 36(4): 454-62.

26. Gunnar MR, Porter FL, Wolf CM, Rigatuso J, Larson MC. Neonatal stress reactivity: Predictions to later emotional temperament. Child Dev 1995; 66(1): 1-13.

27. Johnson BH, Hansen AL, Sollers JJ, Murison R, Julian JF. Heart rate variability is inversely related to cortisol reactivity during cognitive stress. Psychosom Med 2002; 64(1): 128.

28. Sgoifo A, Braglia F, Costoli T, et al. Cardiac autonomic reactivity and salivary cortisol in men and women exposed to social stressors: relationship with individual ethological profile. Neurosci Biobehav Rev 2003; 27(1/2): 179-88. 
29. Murdock KW, LeRoy AS, Fagundes CP. Trait hostility and cortisol sensitivity following a stressor: the moderating role of stress-induced heart rate variability. Psychoneuroendocrinology 2016; 75: 222-7.

30. Castro M, Moreira AC. Análise crítica do cortisol salivar na avaliação do eixo hipotálamo-hipófise-adrenal. Arq Bras Endocrinol Metab 2003; 47(4): 358-67.

31. Abrão AL, Leal SC, Falcão DP. Salivary and serum cortisol levels, salivar alpha-amylase and unstimulated whole saliva flow rate in pregnant and non-pregnant. Rev Bras Ginecol Obstet 2014; 36(2): 72-8.

32. Campos JF, David HMSL. Análise de cortisol salivar como biomarcador de estresse ocupacional em trabalhadores de enfermagem. Rev Enferm UERJ 2014; 22(4): 447-53.

33. Langewitz, W, Ruddel, H, Schachenger H. Reduced parasympathetic cardiac control in patients with hypertension at rest and under mental stress. Am Heart J 1994; 127(1): 122-8.

34. Morino M, Masaki C, Seo Y, et al. Non-randomized controlled prospective study on perioperative levels of stress and dysautonomia during dental implant surgery. J Prosthodont Res 2014; 58(3): 177-83.

Received: May, 22, 2017

Accepted: August, 9, 2017 